\title{
Violation of the Universal Behavior of Membranes Inside Cylindrical Tubes at Nanoscale
}

\author{
E. Perim ${ }^{1}$, A. F. Fonseca ${ }^{1}$, N. M. Pugno ${ }^{2,3,4}$, D. S. Galvao ${ }^{1}$ \\ ${ }^{1}$ Applied Physics Department, State University \\ of Campinas, Campinas-SP, Sao Paulo, Brazil. \\ ${ }^{2}$ Laboratory of Bio-inspired and Graphene Nanomechanics, \\ Department of Civil, Structural and Mechanical Engineering, \\ University of Trento, via Mesiano, 77, 38123 Trento, Italy. \\ ${ }^{3}$ Center for Materials and Microsystems, Fondazione Bruno Kessler, \\ via Sommarive 18, 38123 Povo (Trento), Italy. \\ ${ }^{4}$ School of Engineering and Materials Science, \\ Queen Mary University of London, \\ Mile End Road, London E1 4NS, UK.
}

(Dated: May 4, 2021)

\begin{abstract}
Recently, it was proposed based on classical elasticity theory and experiments at macroscale, that the conformations of sheets inside cylindrical tubes present a universal behavior. A natural question is whether this behavior still holds at nanoscale. Based on molecular dynamics simulations and analytical modeling for graphene and boron nitride membranes confined inside carbon nanotubes, we show that the class of universality observed at macroscale is violated at nanoscale. The precise origins of these discrepancies is addressed and proven to be related to both surface and atomistic effects.
\end{abstract}


Systems exhibiting classes of universalities (where the main properties are material and scale independent[1]) are of great interest since it is possible to reliably predict and generalize the properties of a larger number of structures.

With the advent of nanotechnology, many systems exhibiting counter-intuitive and unusual behaviors have been reported, such as auxetic buckypapers [2, 3], atomic suspended chains [4], and 'exotic' metallic structures that can exist only at nanoscale [5]. Thus, much effort has been devoted to adjust macroscopic models in order to understand the nanoscale effects and the origin of these unusual behaviors.

One important example of these approaches is the macroscopic or continuum modeling of the elastic properties of graphene [6] (single layer graphite). Nevertheless, it was demonstrated [7] that continuum models fail to describe the detailed elastic behavior of single-layer graphene, although they can reliably describe the properties of many-layers systems.

Recently, Romero and co-workers [8], after here named RWC model, published a detailed work on the morphology of coiled elastic sheets inside cylinders. The RWC model is based on classical continuum mechanics and proposes that the observed elastic conformations of the sheets inside the cylinders should exhibit an universal behavior which would be expressed by the $\alpha$ angle formed between the sheets and the tubes (Figure 1(a)). These $\alpha$ angle values should be the same, independently on the size or type of material. These predictions were validated by a series of experimental tests [8].

However, as the RWC model was derived for macroscale systems, it neglects some aspects, which become very important at nanoscale, for instance, the significant stickiness generated by the van der Waals (vdW) forces. Another important aspect not considered in the RWC model was the tube topology aspects at atomistic scale (such as, tube chirality).

In the light of the more recent work of Zhang and collaborators [7], a natural question is whether this so-called "universal" behavior observed at macroscale would hold at nanoscale. In order to address this important question we have carried out fully atomistic molecular dynamics (MD) simulations for nanoscale structural models in association with analytical modeling.

As the RWC model investigated folded sheets placed inside a cylindrical tube, we used graphene (G) [9] and boron nitride (BN) [10] membranes and carbon nanotubes (CNTs) [11] as corresponding nanostructures for sheets and tubes, respectively. The concentrically cylindrical folded (rolled up) G and BN membranes inside the CNTs [12] generate the so-called 
nanoscrolls [13, 14] (Figure 10)).

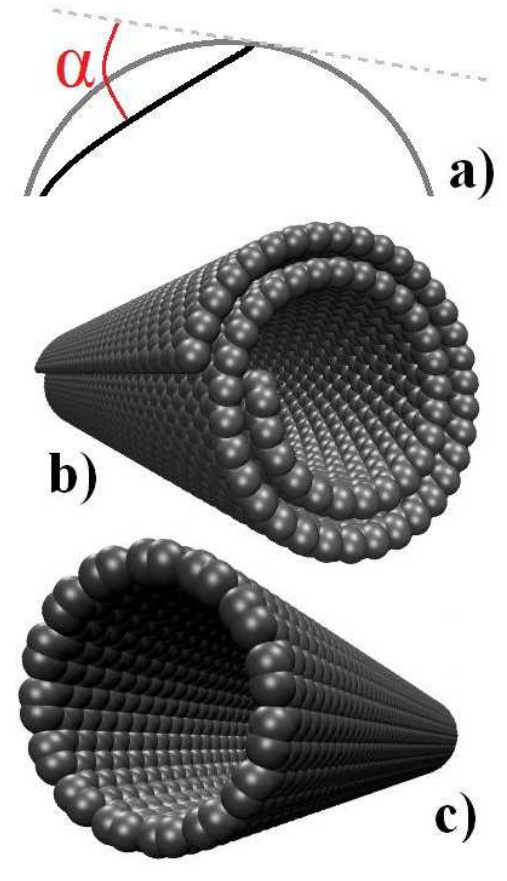

FIG. 1: (Color online) (a) the definition of the $\alpha$ angle. (b) and (c), carbon nanoscroll and carbon nanotube structures, respectively. See text for discussions.

As shown in Figure 1, nanoscrolls are papyrus-like seamlessly wrapped sheets with open ends and can exhibit (depending on the diameter and the materials they are composed of) higher structural stability than their corresponding planar-layered conformations [15], resulting from the attractive vdW forces, which can overcome the elastic ones [16, 17]. Carbon [18] and BN [19, 20] nanoscrolls (CNSs and BNNSs, respectively) have been already experimentally realized. For these nanostructures the vdW forces are of major importance in defining their structural stability and they cannot be neglected as they (in general) are in macroscopic models. In the present work, our models consisted of either carbon or BN membranes coiled/scrolled inside CNTs. Without lack of generality, all G and BN membranes, as well as the used CNTs were of zigzag type [16]. We have considered scrolls formed from membranes with rectangular dimensions of (from 160 up to $320 \AA$ ) by $32 \AA$ which are rolled up as archimedian spirals around the axis along the direction of their smaller side, with constant layer separation of $3.4 \AA$ and inner diameter approximately of $20 \AA$ [16]. The CNT diameter and length were chosen to be compatible with the scroll dimensions, considering the cases of uncompleted rolling up to many-layers scrolls. 
In order to directly contrast our results with the ones from the RWC model (where the tube dimensions do not change), our CNTs were kept frozen in all the simulations.

All MD simulations were carried out using the universal molecular force field (UFF) [21], as implemented in the Materials Studio suite [22]. UFF is a well-known and tested force field and includes bond stretch, bond angle bending, inversion, torsions, rotations and vdW terms. The MD simulations were carried out within the NVE (number of atoms, volume and energy constant) ensemble with convergence criteria of $10^{-5} \mathrm{kcal} / \mathrm{mol}$ for energy and 5 $\times 10^{-3} \mathrm{kcal} \AA / \mathrm{mol}$ for maximum force among atoms, respectively. No explicit charges were used and all atoms were assumed as having partial double bonds and $\mathrm{sp}^{2}$ hybridizations. This approach has been proven to be very effective in the description of mechanical and structural properties of CNTs and scrolls [16, 17].

In order to test the "universal" behavior of the RWC model at nanoscale, we analyzed its critical variable prediction: the angle $\alpha$ formed by the sheets with respect to the tube wall (Figure 1a)). From the RWC model this angle is supposed to have a universal value of $24.1^{\circ}$.

There are many possible configurations for the combination of scrolls and tubes, but we restricted ourselves, due to the lack of space here, to two major cases; (I) the one at which the tube has a diameter large enough for the sheets inside the tubes retain their scrolled conformation and;(II) the case where the sheets lengths are smaller than the tube diameter.

We will start discussing the case (I). In order to preserve their structural stability isolated scrolls must have an inner diameter around $20 \AA[16,17]$. This scenario is analogous to the one treated by the RWC model for $0.26<D / L<0.32$, where $D$ is the tube diameter and $L$ the scroll length.

In Figure 2 we present the obtained scroll morphologies for CNSs and BNNSs, respectively. Accordingly to the RWC model, the scrolls should exhibit two detached regions, for both inner and outer scroll layers but, as we can see from Figure 2(a) and 2(b), this did not occur, either to carbon or BN scrolls, showing that the so-called "universal" behavior predicted by the RWC model is violated at nanoscale, and, consequently, there is no real "universal" behavior for sheets confined inside cylindrical tubes.

For the case (II), where we have sheets that are not larger enough to form complete scrolled structures, we found similar results (see Figure 3(a) and 3(b)). No detachment of the sheets from the tubes were observed and $\alpha$ angle values are again different than the ones 

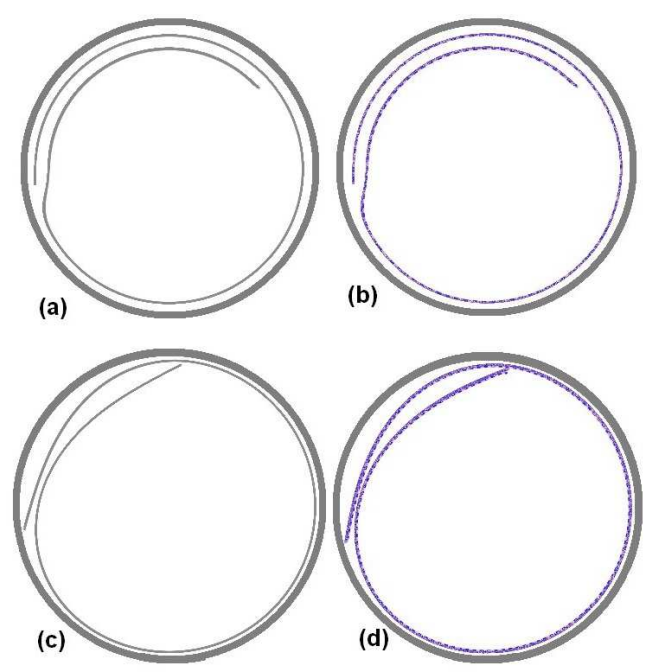

FIG. 2: (Color online) Optimized (a) CNS and (b) BNNS inside a CNT with full vdW interactions; (c) CNS and (d) BNNS with scaled down vdW ones. The measured $\alpha$ angles for cases (c) and (d) were both equal to $28^{\circ}$. See text for discussions.

predicted by the RWC model.
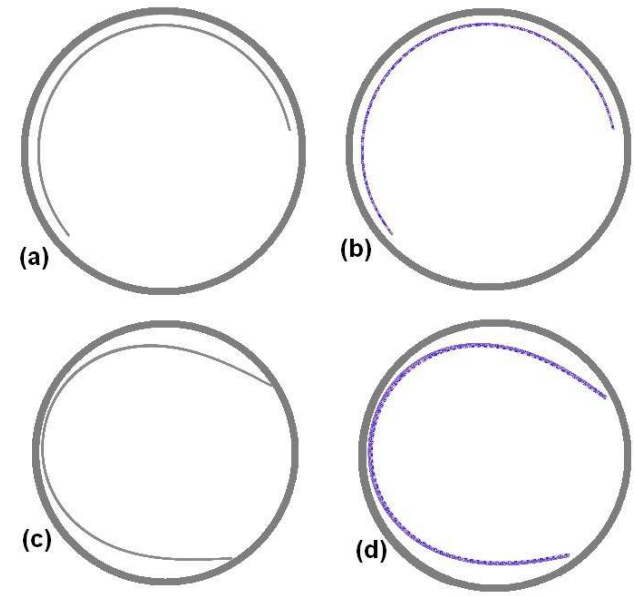

FIG. 3: (Color online) Optimized (a) Unformed scrolled CNS and (b) BNNS inside a CNT with full vdW interactions; (c) CNS and (d) BNNS with scaled down vdW ones. The measured $\alpha$ angles for cases (c) and (d) were both equal to $28^{\circ}$. See text for discussions.

It remains to be elucidated the origin of these apparent discrepancies. As above mentioned, one essential aspect of the models at nano and macro scale are the relative importance of the vdW forces, which were not explicitly taken into account in the RWC model. 
In order to test whether the vdW forces would be in the origin of these discrepancies, we modified the terms related to the vdW forces in our molecular force field and reran the MD simulations. We considered different cases, where the vdW interactions were gradually decreased until the limit situations where the $\mathrm{vdW}$ forces were mainly of repulsive type. This can be done by changing the parameter values in the force field which control the well-depth energy of the Lennard-Jones potential associated with the vdW forces. In our MD simulations these parameters were gradually decreased up to seven orders of magnitude with relation to their standard values. If the vdW forces are the only responsible cause for the $\alpha$ discrepancies, it should be expected that decreasing the vdW forces would make the $\alpha$ values to converge to the macroscopic predicted values.

In Figure 4 we present the results for the carbon scrolls; similar results were obtained for the BN ones. As we can see from the Figure, scaling down the vdW interactions indeed make the $\alpha$ values become closer to the "universal" value predicted by the RWC model. However, these $\alpha$ values vary and, more importantly, they do not converge to the expected macroscopic value. Thus, the vdW contributions alone cannot be associated with the $\alpha$ discrepancies. One possibility is that these discrepancies have a pure atomistic origin, as recently proposed in the literature [7].

In order to test this hypothesis, we have tried a different approach by including a simple modification in the RWC model for the contact angle of the sheets inside the tubes.

We assume that the variation of the $\alpha$ angle with respect to the its macroscopic "universal" value is due to the presence of a pressure variation imposed by the adhesion and a bending stiffness variation due to atomistic effects of a single atomic layer [7].

From the classical elastic solution [24], the pressure at the contact tip is:

$$
p=\frac{B}{2 R^{2} \sin \alpha},
$$

where $B$ is the bending stiffness and $R$ is the tube radius value.

In the presence of adhesion between a tip and a substrate, an adhesive pressure $\left(p_{a}\right)$ proportional to the surface energy $\gamma$ (by a factor of $c / t$, with $c$ constant and $t$ thickness of the layer) naturally emerges. Accordingly [25]:

$$
\frac{p_{a}}{p} \approx \frac{c}{t} \frac{\gamma R^{2}}{B} .
$$

Similarly, considering a variation of pressure $\left(p_{A T}\right)$ as imposed by a variation of the bending 


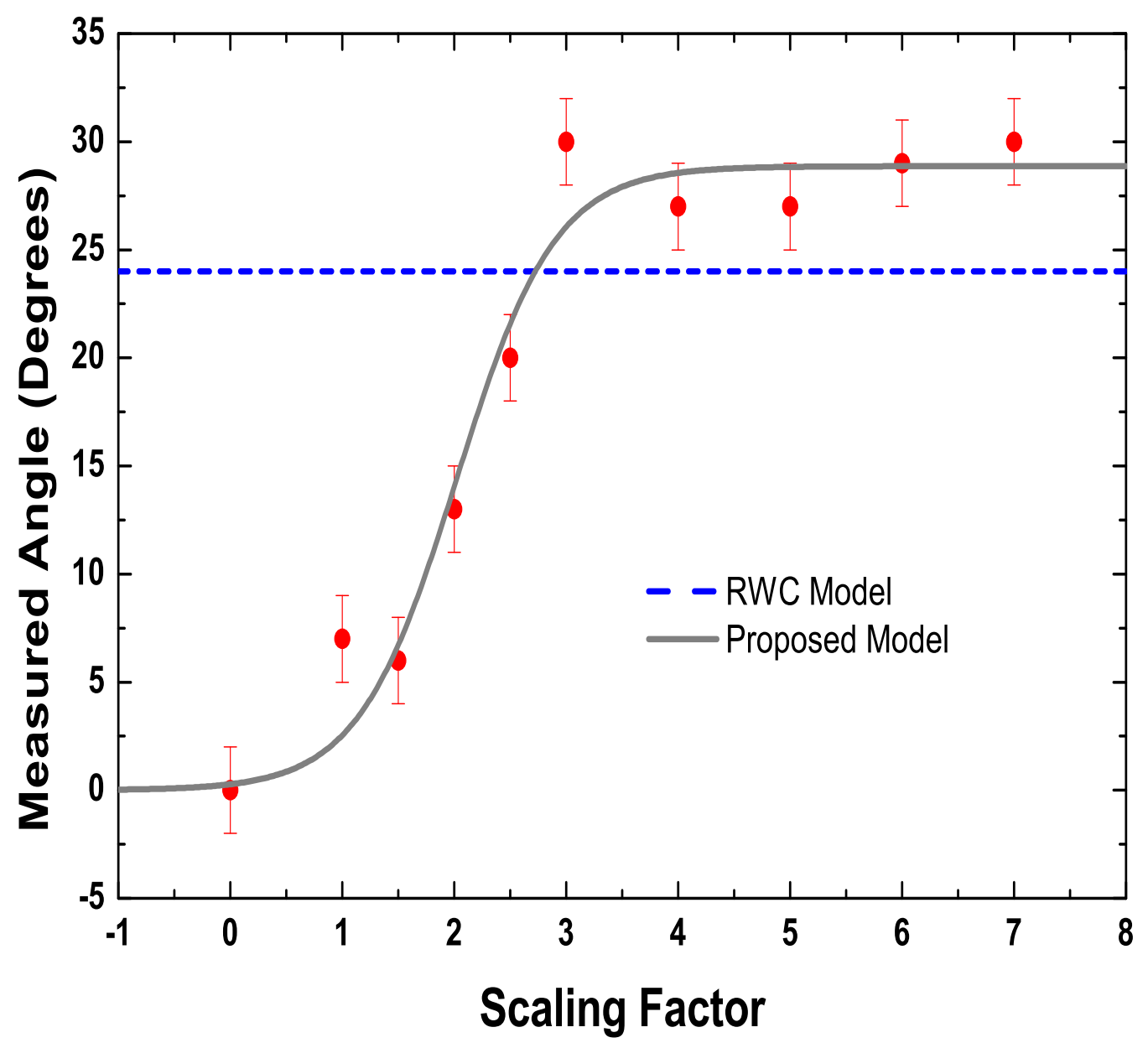

FIG. 4: (Color online) Measure $\alpha$ angle values as a function of the scaled down van der Waals terms. Results obtained for carbon membranes. The Lennard-Jonnes potential well depth $\epsilon(n)$, proportional to the surface energy $\gamma$, is scaled as $\epsilon(n)=\epsilon_{0} / 10^{n}$, where $n$ is the scaling factor. See text for discussions.

stiffness due to atomistic effects [7]:

$$
\frac{p_{A T}}{p} \approx-\frac{\Delta B}{B} .
$$

Imposing: $p^{\prime}=p+p_{a}+p_{A T}=\frac{B}{2 R^{2} \sin \alpha^{\prime}}$, where the $\alpha^{\prime}$ is the nanoscale contact angle, and assuming that the surface energy $\gamma$ is scaled down, we can derive the following correction:

$$
\sin \alpha^{\prime}=\frac{\sin \alpha}{1+c \frac{\gamma R^{2}}{t B}-\frac{\Delta B}{B}} .
$$

When the system is large and atomistic effects negligible, we have, as expected, $\alpha=\alpha^{\prime}$, 
while for a single layer with a fictitiously vanishing surface energy, we have:

$$
\sin \alpha^{\prime}=\frac{\sin \alpha}{1-\frac{\Delta B}{B}} .
$$

Using the data presented in Figure 4 in association with the Equation (4), we can obtain another estimation of the correction values of the bending stiffness variation of a single layer structure, $\Delta B / B$. Through all these assumptions, our corrected model fits very well the data for the angle $\alpha$ as a function of the scaling factor $n$ as shown in Figure 4 . We then obtain an estimate of a variaton of about $15 \%$ on the bending stiffness, which are in excellent agreement with the prediction of Zhang and collaborators [7], thus strongly suggesting that atomistic effects of the single (non-continuous) atomic layer structure are responsible for the $\alpha$ discrepancies.

In summary, we have demonstrated that the so-called "universal" behavior for the conformations of sheets confined inside cylindrical tubes [8], is violated at nanoscale. The origin of the discrepancies between the macro and nanoscale models cannot only be attributed to the relative importance of van der Waals forces, but have also atomistic contributions, as recently predicted by Zhang and collaborators [7].

\section{ACKNOWLEDGMENTS}

This work was supported in part by the Brazilian Agencies CNPq and FAPESP. The authors thank the Center for Computational Engineering and Sciences at Unicamp for financial support through the FAPESP/CEPID Grant \#2013/08293-7. AFF also acknowledges support from FAPESP grant \#2012/10106-8. NMP is supported by the European Research Council, Grants Bihsnam and Replica2 as well as by the European Union, within the Graphene Flagship.

[1] Moon, K. -W. et al. Phys. Rev. Lett. 110, 107203 (2013).

[2] Hall, L. J. et al. Science 320, 504 (2008).

[3] Endo M. et al. Nature 433, 476 (2005).

[4] Rodrigues, V. Sato, F. Galvao, D. S. and Ugarte, D. Phys. Rev. Lett. 99, 255501 (2007).

[5] Machado, L. D. et al. Phys. Rev. Lett. 110, 105502 (2013). 
[6] Cadelano, E. Palla, P. L. Giordano, S. and Colombo L. Phys. Rev. Lett. 102, 235502 (2009).

[7] Zhang, D. -B. Akatyeva, E. and Dumitrica, T. Phys. Rev. Lett. 106, 255503 (2011).

[8] Romero, V. Witten, T. A. and Cerda, E. Proc. R. Soc. A 464, 2847 (2008).

[9] Novoselov, K. et al. Science 306, 666 (2004).

[10] Song, L. et al. Nano Lett. 10, 3209 (2010).

[11] Iijima, S. Nature 354, 56 (1991).

[12] Lavin, J. G. Subramoney, S. Ruoff, R. S. Berber, S. Tomanek, D. Carbon 40, 1123 (2002).

[13] Tomanek, D. Physica B 323, 86 (2002).

[14] Shi, X. Pugno, N. M. Gao, H. Acta Mechanica Solida Sinica 23, 484 (2010).

[15] Shi, X. Pugno, N. M. Cheng, Y. Gao, H. Appl. Phys. Lett. 95, 163113 (2009).

[16] Braga, S. F. et al. Nano Lett. 4, 881 (2004).

[17] Perim, E. and Galvao, D. S. Nanotechnology 20, 335702 (2009).

[18] Viculis, L. M. Mack, J. J. and Kaner, R. B. Science 299, 1361 (2003).

[19] Chen, X. Boulos, R. A. Dobson, J. F. and Raston, C. L. Nanoscale 5, 498 (2013).

[20] Li, X. et al. Adv. Mater. 25, 2200 (2013).

[21] Rappé, A. K. et al. J. Am. Chem. Soc. 114, 10024 (1992).

[22] Materials Studio suite from Accelrys. http://www.accelrys.com.

[23] Legoas, S. B. et al. Phys. Rev. Lett. 90, 055504 (2003).

[24] Landau, L. D. and Lifshitz, E. M. Theory of elasticity, Pergamon Press, New York, 1970.

[25] Pugno, N. M. J. Mech. Phys. Sol. 58, 1397 (2010). 